\title{
In situ Transmission Electron Microscopy Study of Dislocation Emission At Junctions Between $\Sigma=3$ Grain Boundaries In Gold Thin Films
}

\author{
G. Lucadamo and D.L. Medlin \\ Thin Film and Interface Science Dept., Sandia National Laboratories, Livermore, CA 94551
}

The interaction between dislocations and microstructural elements such as grain boundary junctions is an important aspect of deformation and stress relaxation processes in polycrystalline thin films. For instance, in situ TEM investigations of polycrystalline thin films under an applied stress previously showed that dislocations either were emitted or nucleated at triple points between grains and at cusps along grain boundaries [1,2]. These experiments also demonstrated the capability of electron microscopy techniques to provide important information related to dislocation dynamics.

Boundaries in a $\Sigma=3$ misorientation provide one of the simplest cases on which to conduct detailed studies of similar phenomena. Evaporating $\mathrm{Au}$ on $\mathrm{Ge}$ (111) produces a microstructure that is comprised of two domains related by a $180^{\circ}$ rotation about the (111) surface normal $[3,4]$. As a result, only $\Sigma=3\{112\}$ and $\Sigma=3\{111\}$ grain boundaries are present in the film (Fig. 1). The $\{112\}$ boundaries are perpendicular to the substrate and highly faceted. The areas where the domains overlap are twin boundaries. Free-standing, $50 \mathrm{~nm}$-thick Au films for TEM studies were obtained by dissolving the Ge substrate. The in situ TEM experiments were conducted using a JEOL 2010F operated at $200 \mathrm{kV}$ and equipped with a Gatan heating stage.

Planview TEM observations of as deposited films revealed arrays of uniformly spaced dislocations between overlapping domains and near $\{112\}$ boundary junctions (Fig.2). Distinct strain contrast was also visible at sites along the $\{112\}$ boundary preceding the junction. To determine the origin of the arrays, samples were heated to $550^{\circ} \mathrm{C}$ in the microscope and held isothermally. Fig. 3 shows a series of frames captured from a video that revealed the details of the dislocation emission process. Initially, the strain contrast feature in the $\{112\}$ boundary (arrowed) moves towards the facet junction. After passing beyond the junction, a new dislocation segment is formed on the $\{111\}$ twin plane (see Fig.1). The existing and newly emitted segments continue to move away from the facet junction with time. Identical behavior was observed repeatedly in several similar experiments. Threading segments extend through the $\{112\}$ boundaries from the twin plane to the film surfaces. Other experiments indicate that the Burgers vectors are parallel and have the same sign.

The strain contrast features ahead of the junction are secondary grain boundary dislocations that accommodate a small misorientation from exact coincidence. A selected area diffraction pattern that samples both domains reveals spots that are offset (Fig. 4). A possible explanation of the driving force for the emission could be the repulsive interaction between the strain field of the dislocation array and the strain fields of the individual threading segments arising from the dislocations in the $\{111\}$ twin. However, as the dislocations move across the twin, they must also increase in length (i.e., line energy). Calculations indicate that it is energetically favorable for the dislocations to move away from the junction for distances consistent with what was typically observed experimentally $(100-150 \mathrm{~nm})$. A schematic illustration of the overall dislocation/junction geometry determined from these experiments is shown in Fig. 5 [5].

\section{References}

[1] E.A. Stach et al., Mat. Res. Soc. Symp. Proc., Vol. 619, (2000) p. 27.

[2] T.J. Balk et al., Mat. Res. Soc. Symp. Proc., Vol. 673, (2001) p. P2.7.1.

[3] E.W. Dickson and D.W. Pashley, Phil Mag., 7, (1962) 1315.

[4] K.H. Westmacott et al., Phil. Mag. A, 81 (2001) 1547.

[5] This work is supported by U.S. Department of Energy, in part by the Office of Basic Energy Sciences, under contract number DE-AC04-94-A185000. 


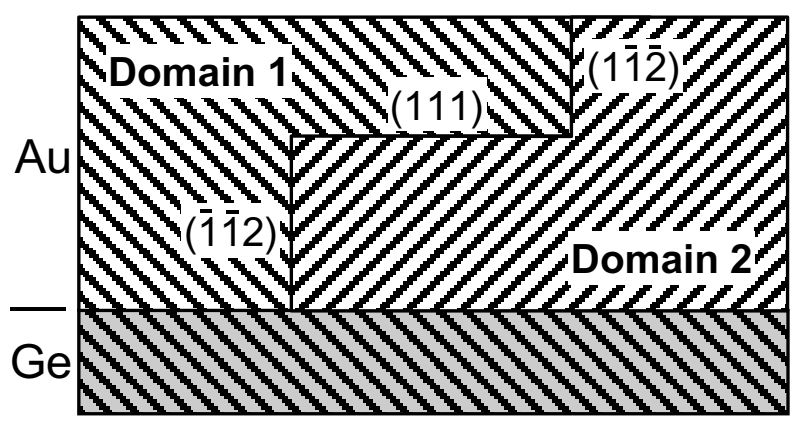

FIG. 1. Schematic illustration of the Au film crystallography as viewed along a $<110>$ zone axis.

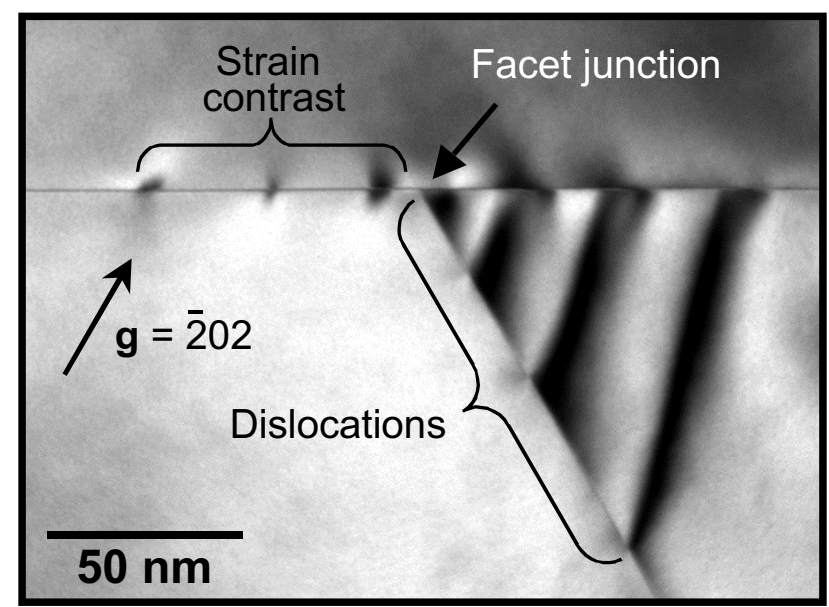

FIG. 2. Bright-field, planview TEM image of dislocation configuration observed around a facet junction.
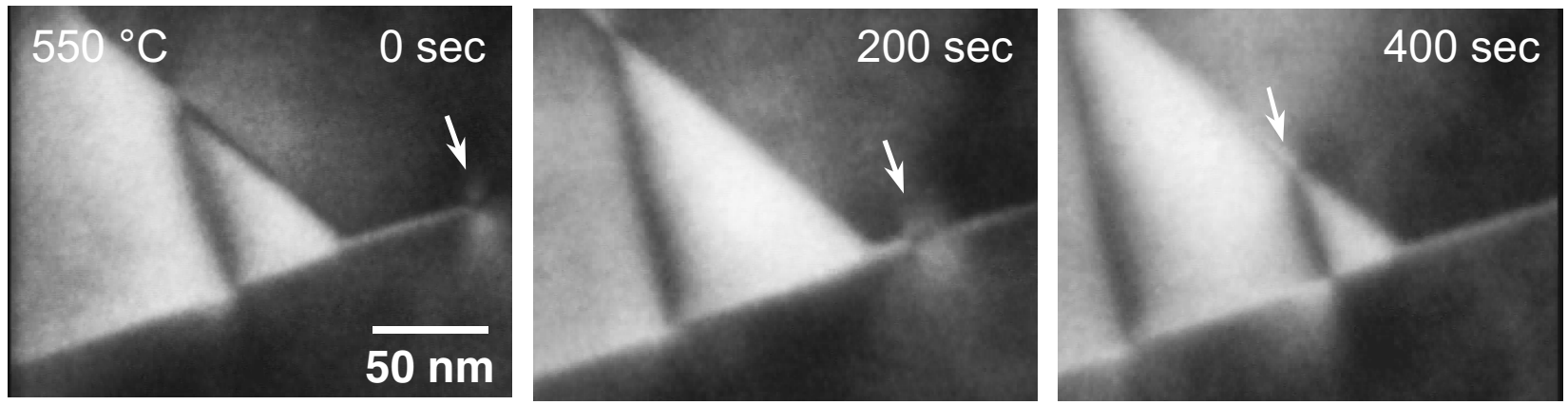

FIG. 3. Series of dark-field images taken from video showing the emission of one dislocation from a $\{112\}$ facet to a $\{111\}$ twin boundary.

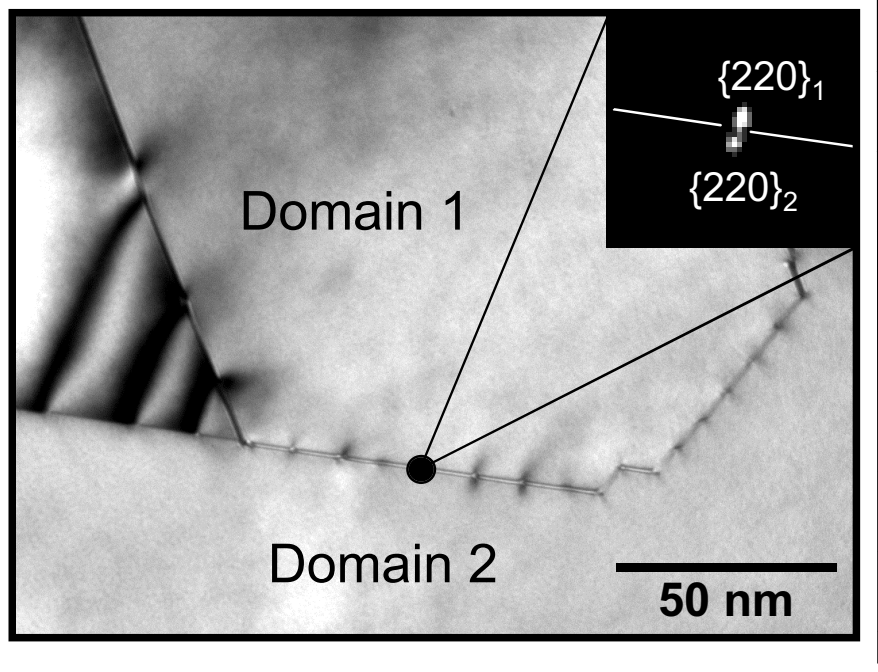

FIG. 4. Bright-field, TEM image and enlarged $\{220\}$ diffraction spot of taken with the selected area aperture positioned over the indicated boundary.

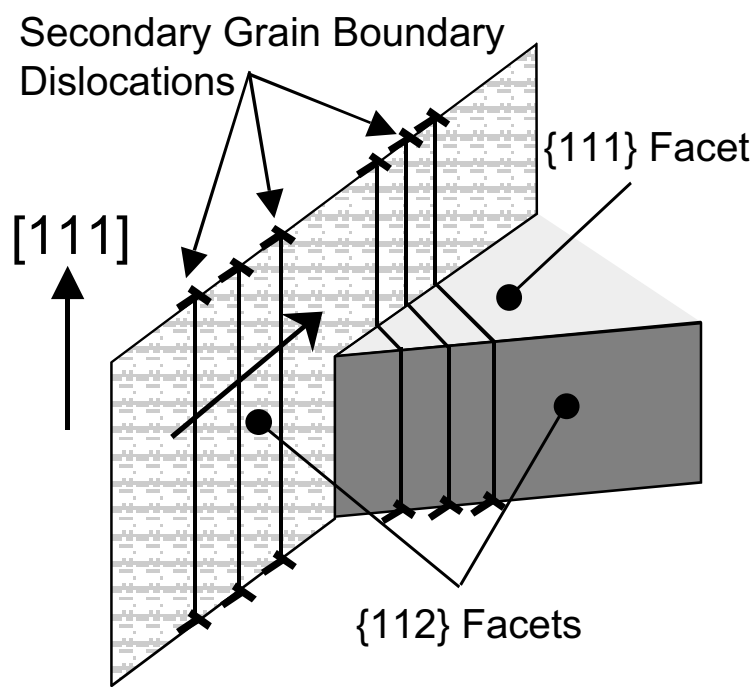

FIG. 5. A schematic illustration depicting the arrangement of dislocations around a facet junction and twin boundary. 